\title{
Special issue on Nature Inspired Cooperative Strategies for Optimisation (NICSO)
}

\author{
N. Krasnogor • G. Nicosia • M. Pavone • \\ D. A. Pelta
}

Published online: 6 January 2010

(C) Springer Science+Business Media B.V. 2010

This special issue collects a selection of the best papers emerging from the 2007 International Workshop on Nature inspired cooperative strategies for optimisation (NICSO 2007).

Nature inspired strategies are being used in ever more widely ranging settings spanning not only computational problem solving (e.g. Neural Networks, Simulated Annealing, Ant Colony Optimisation, etc.) but also on a variety of other disciplines such as chemistry, physics and engineering. Moreover, cooperative strategies are gaining momentum across many computer science disciplines such as machine learning, classification, data mining, and, of course, optimisation.

The NICSO workshop series and its associated publications have been actively helping to advance the state of the art in Nature inspired cooperative strategies for optimisation since its inception in 2006. Some, but not all, of the main themes covered by NICSO include, computational studies in adaptive behavior, amorphous computing, artificial life, ant colonies optimisation, artificial immune systems, swarm intelligence, software selfassembly and self-organisation, evolutionary algorithms, neural networks, etc. as applied to numerical, combinatorial, non linear, dynamic and/or noisy optimisation.

NICSO 2007 received 83 papers, out of which 46 were published in the proceedings under Springer's Studies in Computational Intelligence series. From these 46 papers 12 were invited to the special issue in Natural Computing for which authors were asked to submit extended and improved versions of their works. Submitted papers went through a rigorous two (in some cases three) rounds of additional independent reviewing. Each paper was assigned three or more reviewers. From the 12 papers received, the following five were accepted for this thematic issue.

In "Honey Bees Mating Optimisation Algorithm for Large Scale Vehicle Routing Problems" by Y. Marinakis et al., the authors present a sophisticated multi-hybrid algorithm for the vehicle routing problem. Their algorithm combines two metaheuristics and one heuristic to produce a balanced allocation of effort between local and global search. The Honey Bee Optimisation algorithm that is seeded with an improved variation of

\footnotetext{
N. Krasnogor $\cdot$ G. Nicosia $\cdot$ M. Pavone $(\bowtie) \cdot$ D. A. Pelta

University of Catania, Catania, Italy

e-mail: mpavone@dmi.unict.it
} 
GRASP produce a reliable global search while an expanding neighbourhood search method (related to the well-known variable neighbourhood search) operates as a local search algorithm that adapts its move operator over time. Moreover, the algorithm also uses an improved crossover with adaptive memory as to further maintain diversity and evolvability.

The paper by S. Consoli et al., "Discrete Particle Swarm Optimisation for the Minimum Labelling Steiner Tree Problem" presents a state of the art "Jumping" PSO for combinatorial optimisation. The problem tackled is the Min-Labelling Steiner Tree problem that, given a set of vertices, labels and edges, seeks to produce a minimum spanning tree of the selected (called basic) vertices that utilises the minimum number of labels. This is an important and practical NP-hard problem as it is at the core of many relevant industrial applications such as telecommunications network design and multimodal transportation networks. The authors compare their JPSO method against an exact algorithm, a popular metaheuristic method (Pilot Method) and muti-start technique showing that JPSO is competitive and in many cases superior to the other methods.

G. Gutin et al. in the paper with title "A Memetic Algorithm for the Generalized Traveling Salesman Problem" present a multimeme algorithm for the symmetric and asymmetric graphical travelling salesman problem (GTSP). In the GTSP, one is given a set of cities, partitioned intro groups, and required to produce a tour of minimal length that includes exactly one city from each group. The proposed multimeme algorithm employs the standard evolutionary cycle of crossover, mutation and selection but extended with a battery of local searchers that include, swap moves, k-neighbor swaps, 2-opt tries, direct 2-opts, insertions and cluster optimisation moves. These act cooperatively in tandem and once a given move cannot improve a solution it is removed from the meme set. The results obtained represent the state of the art in asymmetric GTSP and is competitive with the best algorithms for the symmetric version of the problem.

In "Hybrid Functional Petri Nets as MP Systems", Castellini et al. demonstrate a formal equivalence between Hybrid Functional Petri Nets and Metabolic P Systems. Metabolic P Systems are membrane computing's response to the challenge of a rigorous methodology for modelling metabolic processes in living organisms. The equivalence between HFPN and MP systems is exemplified with the lac operon regulatory mechanism in the glycolytic pathway of E. coli.

R.I. Lung et al. in "Evolutionary Swarm Cooperative Optimisation in Dynamic Environments" present a multi-hybrid algorithm for optimisation in dynamic environments. The proposed algorithm amalgamates within a cooperative scheme a Differential Evolution algorithm that manages two different populations and a Particle Swarm Optimisation. The main objective of the DE populations is to produce an effective global search that maintains the populations in a diversified state as to be able to "follow" changes in the fitness landscape without collapsing to poor local optima. On the other hand the PSO component is use to refine solutions thus zooming-in into high quality optima. Several cooperation mechanisms for connecting DE and PSO are proposed and tested.

The guest editors would like to thanks the authors that by submitting papers to the NICSO series are pushing forward the frontiers of Nature inspired cooperative strategies for optimisation. We would also like to wholeheartedly acknowledge the work made by the member of the international programme committee and the reviewers contacted for this special issue for their excellent work in assessing the scientific quality of the submissions. We would also like to thank the Editor in Chief of Natural Computation Dr. G. Rozenberg for welcoming this thematic issue. 
Finally D. A. Pelta acknowledges the support from Projects TIN2008-01948 (Spanish Ministry of Science and Innovation) and P07-TIC-02970 (Andalusian Government), and N. Krasnogor would like to acknowledge the UK EPSRC for funding project EP/ D061571/1. 